\title{
FULLY AUTOMATIC DETECTION OF ANOMALIES ON WHEELS SURFACE USING AN ADAPTIVE ACCURATE MODEL AND HYPOTHESIS TESTING THEORY
}

\author{
Karim Tout, Rémi Cogranne and Florent Retraint \\ LM2S - ICD - UMR 6281 STMR CNRS - Troyes University of Technology (UTT) - Troyes - France. \\ E-mail : \{karim.tout, remi.cogranne, florent.retraint\}@utt.fr \\ Copyright (c)2016 IEEE / EURASIP. \\ Personal use of this material is permitted. \\ Final version available online on ieeexplore.ieee.org. DOI: TBA
}

\begin{abstract}
This paper studies the detection of anomalies, or defects, on wheels' surface. The wheel surface is inspected using an imaging system, placed over the conveyor belt. Due to the nature of the wheels, the different elements are analyzed separately. Because many different types of wheels can be manufactured, it is proposed to detect any anomaly using a general and original adaptive linear parametric model. The adaptivity of the proposed model allows us to describe accurately the inspected wheel surface. In addition, the use of a linear parametric model allows the application of hypothesis testing theory to design a test whose statistical performances are analytically known. Numerical results show the accuracy and the relevance of the proposed methodology.
\end{abstract}

Index Terms - Anomaly detection; Nondestructive testing; Adaptive image model; Hypothesis testing theory.

\section{INTRODUCTION}

During the past decades computer-vision based non-destructive testing have been broadly used for inspection of wide range of "objects" such as fabrics [1, 2], nuclear fuel rods [3] or even food to cite few. However, such systems are usually designed to help and ease an operator who has to do the inspection "manually" which might be subjective and labor intensive. Thus, there is a great need for fully automatic detection methods. However, depending on the inspected object such a detection may be difficult due to the non-anomalous "background".

Prior methods for defect detection using computer-vision can be divided into three categories [1, 4]: 1) Generic methods that are highly flexible since they do not need any prior

The Ph.D thesis of Karim Tout is partially funded by the Conseil Régional de Champagne-Ardenne under Collaborative Research Program. knowledge on the inspected object. Such methods are usually based on tools for image processing and defect enhancement (contrast enhancement, edge detection, etc...) and pattern recognition [2]. 2) Specific methods that are based on ground-truth or examples of a reference [4]. The detection is then merely based on the observed differences between the reference and the inspected image. This approach is efficient but also very sensitive to experimental conditions, such as object position, illumination, etc...3) Methods based on computer-vision and image processing [3, 4], that usually require prior information on the non-anomalous object. Two main approaches have been proposed to introduce statistical prior knowledge: Bayesian and non-Bayesian approaches. Statistical Bayesian approach allows the design of efficient and rather simple methods for anomaly detection. However those methods require 1) that the defect occurs with known prior probability and, 2) that the non-anomalous object is also random with known apriori distribution. Those requirements limit the application of Bayesian methods.

In this paper, it is proposed to design a non-Bayesian method based on an adaptive model of the non-anomalous wheel image, referred to as the "background". The model is sufficiently accurate and flexible for inspection of a wide range of wheels.

The main contributions of the present paper are the following: 1) An adaptive statistical model is proposed to represent the imaged wheels. This model only requires knowledge of geometry which makes the proposed method fully-automatic and applicable to a wide range of wheels.

2) The proposed model is accurate enough, to ensure high detection performance and computationally simple, for realtime application.

3) The statistical properties of the method are explicitly provided. Hence, the operator can, for instance, prescribe a false-alarm probability easily and can know which type of anomalies can be detected with which probability. 

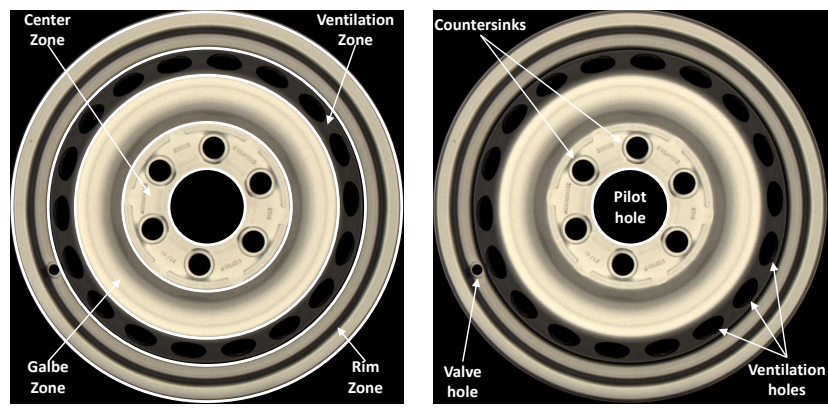

Fig. 1: Description of the different elements of a wheel.

The present paper is organized as follows. Section 2 describes the different elements of a wheel. Then, in Section 3 , the proposed adaptive model of the wheels is detailed. Section 4 presents the proposed statistical method for anomaly detection and studies its statistical properties. Finally Section 5 presents numerical results and Section 6 concludes the paper.

\section{WHEEL'S ELEMENTS DETECTION}

The face of the wheel is a complicated surface to inspect because each wheel is made of different parts (zones). The methodology proposed in this paper is applied on each zone separately, hence the first step is to split the image of a wheel into those different parts, see Fig. 1a. Besides, a wheel also contains some key elements among which the most important are the pilot hole, the countersinks, the valve hole and the ventilation holes, see Fig. 1b. Those elements must be localized for the two following reasons 1) because those are used to perform a geometrical re-adjustment, which can be considered as a self-calibration and 2) those element must be taken into account into the detection of anomalies. Hence, let us first briefly describe how those key elements are detected. It is important to note that, the design of each wheel is known, thus the parameters describing the geometry of inspected wheels, especially the key elements mentioned above, are known.

The pilot hole, the countersinks, and the valve hole, all have a circular shape. Thus, their detection and localization is carried out using the Circular Hough Transform (CHT). This is one of the most robust methods for circular shape detection [5]. In our case, the CHT is also computationally very efficient since it is applied on a limited search area and, because the radius of each element is known, only two unknown parameters have to be found: the coordinates $\left(x_{0}, y_{0}\right)$ of the center of each element.

The last and most complicated step is the detection of the ventilation holes. Those elements exhibit a wide range of different designs for aesthetic reasons but also to reduce the weight of the wheel. This high variety in shapes of the ventilation holes explain the choice of the active contour models, or snakes, for their detection [6]. Such models have been exten-

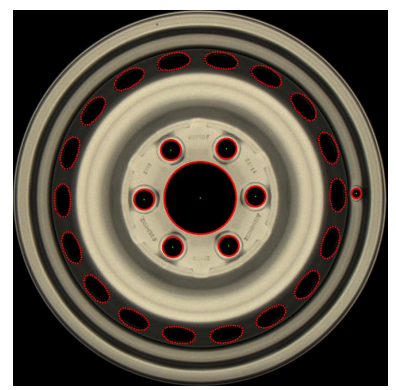

Fig. 2: Illustration of results for detection of all the holes

sively used in image segmentation in order to detect complex geometrical forms. The final result of the detection procedure to localize all those elements is shown in Fig. 2

\section{ADAPTIVE MODEL OF THE WHEEL}

Once the key elements described above are localized, one can split the wheel within several areas, see Fig. 1a.

\subsection{Background model}

For each inspected area, an original adaptive model is applied to subtract the anomaly-free image of the wheel. In fact this "background" acts here as a nuisance as it has no interest for defect detection while must be carefully taken into account. The present paper proposes to use a parametric linear model to represent the background. Such a model has, indeed, indisputable advantages: it is simple and, hence, usually computationally efficient and can be used within the well-founded statistical theory of invariance.

Due to space limitation, the rest of this paper focuses on the galbe zone. For clarity and simplicity, the galbe zone is unfolded to a rectangular image, denoted $\mathbf{Z}=\left\{z_{m, n}\right\}$, of size $M \times N$. The inspected area corresponding to image $\mathbf{Z}$ is split into non-overlapping small blocks of size $w \times h$ (for width and height resp.). Let us also denote $\mathbf{z}_{k}$ the $k$-th block from $\mathbf{Z}$. Because the image $\mathbf{Z}$ is corrupted with additive Gaussian noise, each block $\mathbf{z}_{k}$ can be modeled as:

$$
\mathbf{z}_{k} \sim \mathcal{N}\left(\boldsymbol{\mu}_{k}, \boldsymbol{\Sigma}_{k}\right)
$$

where $\mathcal{N}$ represents the Gaussian distribution, which expectation $\boldsymbol{\mu}_{k}$ and covariance matrix $\boldsymbol{\Sigma}_{k}$. The idea of a linear model is to represent the expectation as a sum of non-anomalous "basis vector" $\boldsymbol{\mu}_{k}=\mathbf{H x}_{k}$. However, the present paper uses an original model for the background expectation, which is adaptive in the sense that the basis vectors $\mathbf{H}$ are actually changed to model better each block. This paper also uses a realistic noise model, discussed in subsection 3.2

The proposed parametric background model is based on 
the following two dimensional algebraic polynomial:

$$
f(x, y)=\sum_{i=0}^{d_{x}} \sum_{j=0}^{d_{y}} c_{i, j} x^{i} y^{j}
$$

with $d_{x}$ and $d_{y}$ the degree of the polynomial.

For simplicity, the block $\mathbf{z}_{k}$ is represented as a vector, reading pixels lexicographically. The same applies for the (discrete) coordinates, denoted as $\mathbf{x}$ and $\mathbf{y}$, and for the coefficients $c_{i, j}$ of the polynomial (2), denoted as vector $\mathbf{c}_{k}$ of $\left(d_{x}+1\right) \times\left(d_{y}+\right.$ $1)$ dimension. Denoting as matrix $\mathbf{F}$ the polynomial model, which is detailed in [3, 7, 8] but not in this paper due to space limitation, the model of the background (2) can be written as:

$$
\boldsymbol{\mu}_{k}=\mathbf{F} \mathbf{c}_{k},
$$

The model (1) - (3) is simple and efficient enough for several applications [3, 7, 8]. However, the non-anomalous background of a wheel is too much complex, with multiple light reflection and artifacts, to be represented with a simple polynomial model. To tackle this difficulty it is proposed in this paper to design an adaptive model, for which the matrix $\mathbf{F}$ changes to take into account the specificity of each block. For this purpose, it is proposed to exploit the circularity of the wheel. Roughly speaking, along the angle, pixels should share similar profiles. The design of an adaptive linear model based on this idea is done using the Principal Component Analysis (PCA). In fact, without subtracting the empirical mean, the first principal components account for the most significant components (eigenvector with highest eigenvalues) of the signal and can be added to the model (3). One can note that sparse dictionary learning methods are also efficient to this purpose but we observed that the PCA provides good results for a much lower computational complexity.

Let us denote $\mathbf{P}_{k}$ the part of the first principal components that corresponds to the locations along the rows of the extracted block $\mathbf{z}_{k}$. With the addition of the adaptive part due to the few first principal components, the proposed model for representing the background, that is the expectation of the block $\mathbf{z}_{k}[1]$, can be written as :

$$
\boldsymbol{\mu}_{k}=\mathbf{H}_{k} \mathbf{d}_{k}
$$

where the matrix $\mathbf{H}_{k}$ is given by the concatenation:

$$
\mathbf{H}_{k}=\left(\mathbf{F} \mid \mathbf{P}_{k}\right) .
$$

Similarly the weighting vector $\mathbf{d}_{k}$ represents the contribution of those different basis vectors.

Roughly speaking, the principal components allow us to model with high accuracy the complexity of the background, and the polynomial part models the remaining part that changes from block to block.

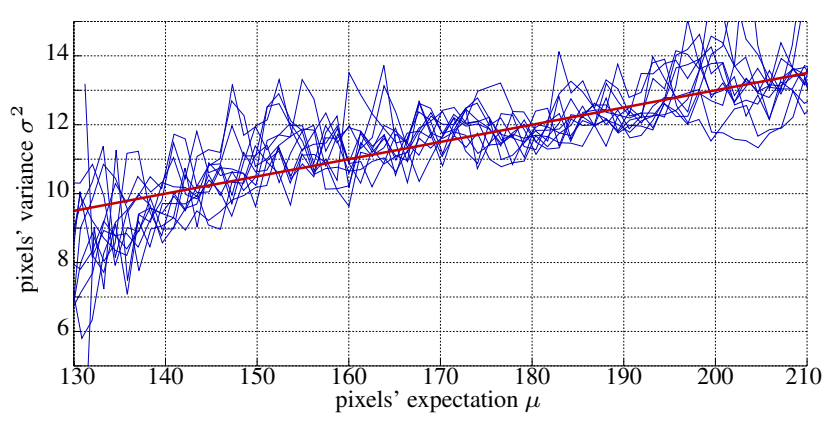

Fig. 3: Illustration of the noise model showing, for several images, pixels' variance as a function of expectation.

\subsection{Noise model}

A usual model for the noise corrupting raw images (that are not processed) can be obtained by considering separately the shot noise, due to Poissonian process of photo-counting, and the various electronic noises. While the variance of the Poissonian process equals its expectation, the electronic noises are well modeled as a Gaussian random variable with constant variance [9, 10]. Due to the very high number of counted photons, the Poissonian process can be approximated as a Gaussian distribution [9, 10]. Hence, the value of pixel at location $(m, n)$ can be modeled as a Gaussian random variable whose variance is given by:

$$
\sigma_{m, n}^{2}=a \mu_{m, n}+b
$$

These parameters $(a, b)$ of the heteroscedastic noise model (5) only depend on the imaging system and acquisition settings. Thus, because neither the camera nor the acquisition settings change, parameters $(a, b)$ remain constant for all pixels from all the images of wheels. After camera calibration, this paper assumes that the noise model parameters $(a, b)$ are known.

The model of the noise (5) allows us to take into account the variance of each pixel in the ensuing statistical test, to improve its accuracy [10,11]. An example of this model (5) is illustrated in Fig. 3. This Figure shows the variance of pixels as a function of estimated expectation from the same pixels highlighting the linear relation between them (5).

Since the pixels do not share the same variance, the estimation of expectation of block $\mathbf{z}_{k}$ is made difficult. To tackle this estimation problem without applying a time-consuming optimization algorithm, a two step approach is proposed in this paper. A first estimation of pixels expectation and variance is obtained with a simple Least-Square (LS):

$$
\left\{\begin{array}{l}
\widetilde{\boldsymbol{\mu}}_{k}^{\mathrm{ls}}=\mathbf{H}_{k}\left(\mathbf{H}_{k}^{\mathrm{T}} \mathbf{H}_{k}\right)^{-1} \mathbf{H}_{k}^{\mathrm{T}} \mathbf{z}_{k}, \\
\widetilde{\boldsymbol{\Sigma}}_{k}^{\mathrm{ls}}=\mathbf{I}_{w \times h} \times\left(a \widetilde{\boldsymbol{\mu}}_{k}^{\mathrm{ls}}+b\right),
\end{array}\right.
$$

where $\mathbf{I}_{w \times h}$ denotes identity matrix of size $w \times h$. This rough estimation of the covariance is thus re-used to update the estimation of the expectation using the well-known Weighted 
Least-Square (WLS) given by:

$$
\left\{\begin{array}{l}
\widetilde{\boldsymbol{\mu}}_{k}=\mathbf{H}_{k}\left(\mathbf{H}_{k}^{\mathrm{T}} \widetilde{\boldsymbol{\Sigma}}_{k}^{\mathrm{ls}^{-1}} \mathbf{H}_{k}\right)^{-1} \mathbf{H}_{k}^{\mathrm{T}} \widetilde{\boldsymbol{\Sigma}}_{k}^{\mathrm{s}^{-1}} \mathbf{z}_{k}, \\
\widetilde{\boldsymbol{\Sigma}}_{k}=\mathbf{I}_{w \times h} \times\left(a \widetilde{\boldsymbol{\mu}}_{k}+b\right) .
\end{array}\right.
$$

It is of course possible to continue this procedure. It has been observed that this two-steps method is a good trade-off between accuracy and computational time.

\section{STATISTICAL DETECTION OF ANOMALIES}

As described above, see Eq. (4), when there is no anomaly, the expectation of block $\mathbf{z}_{k}$ is given by $\mathbf{H}_{k} \mathbf{d}_{k}$. On the opposite, when an anomaly is present in the block $\mathbf{z}_{k}$, its expectation is modified and is modeled by $\mathbf{H}_{k} \mathbf{d}_{k}+\boldsymbol{\theta}_{k}$ with $\boldsymbol{\theta}_{k}$ the expectation of the anomaly. For simplicity, it is assumed in this paper that the presence of the anomaly has no effect on the variance.

Hence the goal of anomaly detection problem studied in the present paper is to decide between these two following composite hypotheses:

$$
\left\{\begin{array}{l}
\mathcal{H}_{0}:\left\{\mathbf{z}_{k} \sim \mathcal{N}\left(\mathbf{H}_{k} \mathbf{d}_{k}, \boldsymbol{\Sigma}_{k}\right), \forall k \in\{1, \ldots, K\}\right\} \\
\mathcal{H}_{1}:\left\{\mathbf{z}_{k} \sim \mathcal{N}\left(\mathbf{H}_{k} \mathbf{d}_{k}+\boldsymbol{\theta}_{k}, \boldsymbol{\Sigma}_{k}\right), \forall k \in\{1, \ldots, K\}\right\},
\end{array}\right.
$$

with of course, $\boldsymbol{\theta}_{k} \neq 0$ for some $k$.

Formally, a statistical test $\delta$ is a mapping $\delta: \mathbb{R}^{w . h} \mapsto$ $\left\{\mathcal{H}_{0} ; \mathcal{H}_{1}\right\}$. The false alarm probability of a test is defined as:

$$
\alpha_{0}(\delta)=\mathbb{P}_{\mathcal{H}_{0}}\left(\delta(\mathbf{Z})=\mathcal{H}_{1}\right)
$$

where $\mathbb{P}_{\mathcal{H}_{0}}$ denotes the probability under hypothesis $\mathcal{H}_{0}$. Conversely, the power function of a test $\delta$ is defined as:

$$
\beta\left(\delta ; \boldsymbol{\theta}_{k}\right)=\mathbb{P}_{\mathcal{H}_{1}}\left(\delta(\mathbf{Z})=\mathcal{H}_{1}\right) .
$$

Note that the power $\beta\left(\delta ; \boldsymbol{\theta}_{k}\right)$ depends on the anomaly $\boldsymbol{\theta}_{k}$. In this paper it is proposed to apply the invariance principle to remove the nuisance parameters $\mathbf{H}_{k} \mathbf{d}_{k}$ and to design a Uniformly Best Constant Power (UBCP) test [12]. To this end, the observations $\mathbf{z}_{k}$ are projected onto the orthogonal complement of the sub-space spanned by the columns of $\mathbf{H}_{k}$ by using the projector:

$$
\mathbf{P}_{\mathbf{H}_{k}}^{\perp}=\mathbf{I}_{w \times h}-\left(\mathbf{H}_{k}\left(\mathbf{H}_{k}^{\mathrm{T}} \widetilde{\boldsymbol{\Sigma}}_{k}^{-1} \mathbf{H}_{k}\right)^{-1} \mathbf{H}_{k}^{\mathrm{T}}\right) \widetilde{\boldsymbol{\Sigma}}_{k}^{-1} .
$$

However, because the variance is not constant over all the pixels, it is necessary to normalize the "residuals" by dividing each residual by its standard deviation. Those normalized residuals can be written as follows:

$$
\mathbf{r}_{k}=\widetilde{\mathbf{\Sigma}}_{k}^{-1 / 2}\left(\mathbf{P}_{\mathbf{H}_{k}}^{\perp} \mathbf{z}_{k}\right) .
$$

It is then easy to establish [12, 3] that the norm of the normalized "residuals" $\mathbf{r}_{k}$ follows the distribution

$$
\left\|\mathbf{r}_{k}\right\|_{2}^{2} \sim \begin{cases}\chi_{\Upsilon}^{2}(0), \forall k \in\{1, \ldots, K\} & \text { under } \mathcal{H}_{0} \\ \chi_{\Upsilon}^{2}\left(\varrho_{k}\right), \forall k \in\{1, \ldots, K\} & \text { under } \mathcal{H}_{1},\end{cases}
$$

where $\chi_{\Upsilon}^{2}\left(\varrho_{k}\right)$ denotes the non-central $\chi$-squared distribution with $\Upsilon=w \times h-p$ degree of freedom, here $p$ denotes the number of columns of $\mathbf{H}_{k}$, and the non-central parameter $\varrho_{k}$ under hypothesis $\mathcal{H}_{1}$ is given by :

$$
\varrho_{k}=\left\|\widetilde{\boldsymbol{\Sigma}}_{k}^{-1 / 2} \mathbf{P}_{\mathbf{H}_{k}}^{\perp} \boldsymbol{\theta}_{k}\right\|_{2}^{2} .
$$

Here $\varrho_{k}$ denotes the "anomaly-to-noise" ratio [3] and is essential to define how detectable the anomaly is.

Based on the residuals $\mathbf{r}_{k}$ and their distribution, see Eq. (10), the UBCP test can be written as follows

$$
\delta= \begin{cases}\mathcal{H}_{0} & \text { if }\left\|\mathbf{r}_{k}\right\|_{2}^{2} \leq \tau \\ \mathcal{H}_{1} & \text { if }\left\|\mathbf{r}_{k}\right\|_{2}^{2}>\tau\end{cases}
$$

where, in order to guarantee the false-alarm probability $\alpha_{0}$, the decision threshold $\tau$ is set as follows:

$$
\tau=F_{\chi_{\Upsilon}^{2}}^{-1}\left(1-\alpha_{0} ; 0\right)
$$

where $F_{\chi_{\Upsilon}^{2}}\left(x, \varrho_{k}\right)$ and $F_{\chi_{\Upsilon}^{2}}^{-1}\left(x, \varrho_{k}\right)$ resp. represent the noncentral $\chi^{2}$ cumulative distribution function with non-centrality parameter $\varrho_{k}$ and its inverse.

Similarly the power function of the test is given by:

$$
\beta\left(\delta, \boldsymbol{\theta}_{k}\right)=F_{\chi_{\Upsilon}^{2}}\left(\tau, \varrho_{k}\right) .
$$

One can note from the previous results, Eq. (13)-(14), two important things. First, the threshold $\tau$ depends only on the false-alarm probability $\alpha_{0}$ and is thus constant for all the blocks. Second, the detectability of the anomaly depends only on the "anomaly-to-noise ratio" $\varrho_{k}$. More precisely, Eq. (11) shows that it is crucial for the model to represent the expectation of $\mathbf{z}_{k}$ with high accuracy but also ensure that the anomaly $\boldsymbol{\theta}_{k}$ remains in the residuals (orthogonal complement of $\mathbf{H}_{k}$ ).

\section{EXPERIMENTS AND RESULTS}

Because of the space limitation, only a limited number of results can be presented. To show the accuracy of the proposed adaptive model, Fig. 4 presents two examples of galbe zones, with typical defects intended to be detected. Note that the defects are highlighted with red circles and that we picked, on purpose, defects which are difficult to see from naked eyes.

First, Fig. $4 \mathrm{c}$ and $4 \mathrm{~d}$ show the normalized residuals $\mathbf{r}_{k}$, see Eq. 99, for the second example of anomaly. The image from Fig. $4 \mathrm{c}$ has been obtained using the proposed adaptive model over blocks with size $h=23$ (height) and $w=50$ (width). The degree of the polynomial used is $d_{y}=5$ (along the height) and $d_{x}=1$ (along the width) and the number of Principal Components added to this model is 3 . The defect is clearly visible which confirms that the proposed adaptive model has the ability to describe with accuracy the background while preserving the anomaly in the "residuals". 


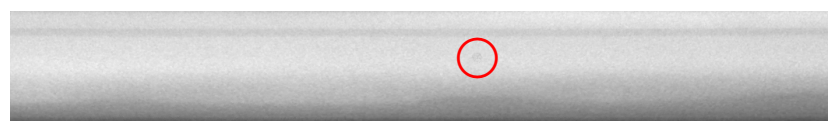

(a) First example of circular shape anomaly.

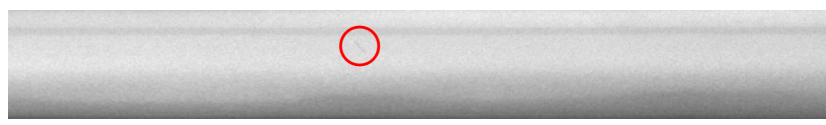

(b) Second example of rectilinear shape anomaly.

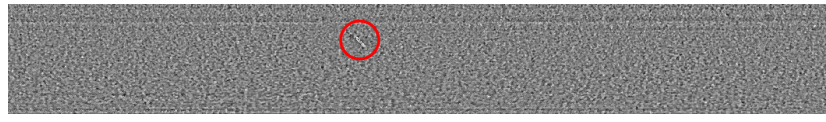

(c) Normalized residuals obtained from Fig. $4 \mathrm{~b}$ with the proposed model.

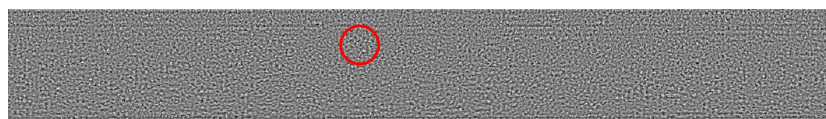

(d) Normalized residuals obtained from Fig. $4 \mathrm{~b}$ with wavelet decomposition.

Fig. 4: Example of typical anomalies it is aim at detecting.

Contrast this result with the image from Fig. 4d which shows the same normalized residuals $\mathbf{r}_{k}$ obtained using the usual wavelet decomposition for estimating pixels' expectation. The wavelet used in this Figure is the Symlet 2, with three levels of decomposition and soft thresholding of the coefficients. With such a generic approach, it is very difficult, for any background and for any anomaly, to model accurately the background while preserving the anomaly in the residuals.

Second, it is wished to show the relevance of the proposed statistical test and the accuracy of the theoretical results. The first example of defect, see Fig. 4a. has been used to perform a Monte-Carlo simulation on 1000 images. Because it is hardly possible to obtain many images with similar anomalies, we picked randomly a set of 1000 images without any defect on which the anomaly has been superimposed. Fig. 5 presents the empirical distribution of the normalized residuals norm $\left\|\mathbf{r}_{k}\right\|_{2}^{2}$, see Eq. (12). One can note from Fig. 5 that the two hypothesis are separable without any classification error.

Beside, Fig. 5 also compares the empirical distribution under $\mathcal{H}_{0}$ with the theoretical one $[10$. A non-negligible discrepancy can be observed which may be explained by two reasons. First, the estimation of pixels expectation has a non-zero variance which is not yet taken into account in the proposed test. Second, the proposed adaptive model, though efficient, is not perfect and, hence, may sometime put a small part of the nonanomalous background among the residuals.

\section{CONCLUSION}

This paper studies the problem of fully automatic anomaly detection on wheels. The proposed method analyzes the different zones of a wheel separately. The main originalities of the proposed approach are the use of an adaptive linear model for the non-anomalous background and the heteroscedastic noise model. The proposed adaptive model is simple, accu-

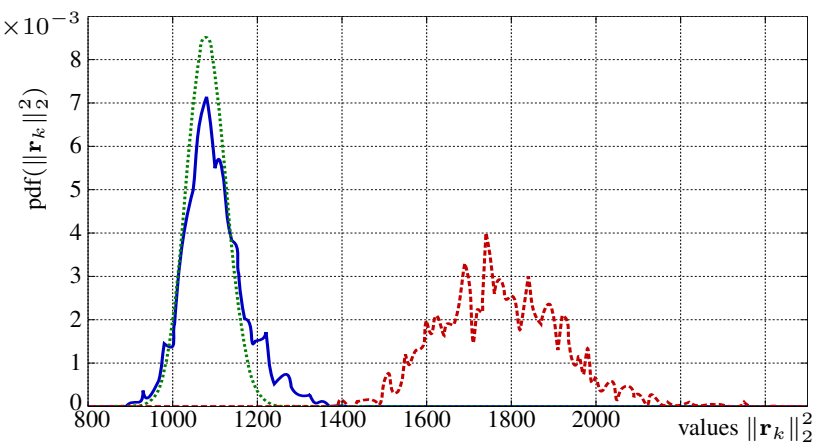

Fig. 5: Empirical and theoretical distributions of the proposed test over 1000 of images with and without anomaly.

rate and preserves the anomaly within the residuals. Because the adaptive model is linear, it can be used easily with theory of hypothesis testing taking into account the noise model. The proposed method allows a fully-automatic detection scheme in real time. Numerical results show the high accuracy of the model and the relevance of the statistical test.

\section{REFERENCES}

[1] A. Kumar, "Computer-vision-based fabric defect detection: a survey," Industrial Electronics, IEEE Trans. on, vol. 55, no. 1, pp. 348-363, 2008.

[2] R. Stojanovic, P. Mitropulos, C. Koulamas, Y. Karayiannis, S. Koubias, and G. Papadopoulos, "Real-time vision-based system for textile fabric inspection," RealTime Imaging, vol. 7, no. 6, pp. 507-518, 2001.

[3] R. Cogranne and F. Retraint, "Statistical detection of defects in radiographic images using an adaptive parametric model,' Signal Processing, vol. 96, Part B, pp. 173 189, March 2014.

[4] D. Mery, T. Jaeger, and D. Filbert, "A review of methods for automated recognition of casting defects," J. Brit. Inst. Non-Destructive Testing, vol. 44, no. 7, pp. 428436, 2002.

[5] J. Illingworth and J. Kittler, "A survey of the hough transform," Computer vision, graphics, and image processing, vol. 44, no. 1, pp. 87-116, 1988.

[6] M. Kass, A. Witkin, and D. Terzopoulos, "Snakes: Active contour models," Int. journal of computer vision, vol. 1, no. 4, pp. 321-331, 1988.

[7] R. Cogranne and F. Retraint, "An asymptotically uniformly most powerful test for LSB matching detection," Information Forensics and Security, IEEE Trans. on, vol. 8, no. 3, pp. 464-476, March 2013.

[8] V. Sedighi, R. Cogranne and J. Fridrich, "ContentAdaptive Steganography by Minimizing Statistical De- 
tectability," Information Forensics and Security, IEEE Trans. on, vol. 11, no. 2, pp. 221-234, 2016.

[9] A. Foi, M. Trimeche, V. Katkovnik, and K. Egiazarian, "Practical poissonian-gaussian noise modeling and fitting for single-image raw-data," Image Processing, IEEE Trans. on, vol. 17, no. 10, pp. 1737-1754, Oct. 2008.

[10] T. H. Thai, R. Cogranne, and F. Retraint, "Camera model identification based on the heteroscedastic noise model," Image Processing, IEEE Trans. on, vol. 23, no. 1, pp. 250-263, 2014.

[11] T. H. Thai, F. Retraint, and R. Cogranne, "Statistical detection of data hidden in least significant bits of clipped images," vol. 98, pp. 263 - 274, May 2014.

[12] M. Fouladirad, L. Freitag, and I. Nikiforov, "Optimal fault detection with nuisance parameters and a general covariance matrix," Intl Journal of Adaptive Control and Signal Processing, vol. 22, no. 5, pp. 431-439, 2008. 